\title{
Preoperative Genetic Testing and Personalized Medicine: Changing the Care Paradigm
}

\author{
Rodney A. Gabriel ${ }^{1,2}$ • Jesse M. Ehrenfeld ${ }^{3} \cdot$ Richard D. Urman $^{4}$
}

Published online: 17 October 2017

(C) Springer Science+Business Media, LLC 2017

Personalized medicine is the concept of tailoring pharmacotherapy to an individual patient based on their predicted response to that therapy $[1,2]$. Its application to perioperative medicine is novel and has much potential for growth. Pharmacogenomics (PGx) is the study of inherited differences in drug metabolism. It is a key component of personalized medicine as a better understanding of the association of an individual's genome and response to therapy may potentially improve outcomes [1,2]. Over the last decade, several studies have demonstrated an association of genotype with efficacy of various medications applicable to the surgical setting [3-8]. Furthermore, in one recent study when PGx results were integrated into an Enhanced Recovery After Surgery (ERAS) pathway for abdominal surgery, there was an improvement in analgesia and decrease in opioid consumption [9].

Although perioperative PGx testing holds much promise, we have not yet been able to achieve successful implementation across our medical systems and into daily practice. In order to obtain more widespread adoption of perioperative personalized medicine, there needs to be: 1) more substantial evidence that perioperative outcomes improve with PGx; 2)

This article is part of the Topical Collection on Patient Facing Systems

Richard D. Urman

rurman@bwh.harvard.edu

1 Department of Anesthesiology, University of California, San Diego, San Diego, CA, USA

2 Department of Biomedical Informatics, University of California, San Diego, San Diego, CA, USA

3 Vanderbilt University Medical Center, Nashville, TN 37232, USA

4 Department of Anesthesiology, Perioperative, and Pain Medicine, Brigham and Women's Hospital/Harvard Medical School, Boston, MA 02115, USA easy-to-access and -understand information technology interfaces that display the relevant PGx information for a patient; and 3) systems put into place that allow PGx to easily fit into regular clinical workflow.

The first point requires the healthcare researcher community to utilize PGx to create the evidence based from highquality prospective studies. The second and third points will require adequate institutional informatics support and infrastructure. Several genes have already been implicated in perioperative medication metabolism [1]. Analysis and presentation of the results of individual mutations to these genes can theoretically be long and daunting. One option is to present a list of common perioperative medications that have some prevalence of genetic risk and classify each medication based on the estimated degree of metabolism that patient has for it (i.e. ultra-metabolizer, extensive metabolizer, slow metabolizer, etc) (Fig. 1).

Finally, to facilitate clinical adoption, PGx screening results should be transferred directly into electronic health record (EHR) systems and seamlessly integrated into the patients' anesthesia and postoperative record. Important components of a successfully integrated PGx program into an EHR include: 1) customized report generation unique to each health institution; 2) tailored data analytics; 3) data presentable for research studies; 4) easy-to-use provider-facing interface in the EHR explaining the key salient results (and recommendations) for a patient as well as a separate section with more granular detail; and 5) EHR-integrated real-time alerts warning providers regarding genetic risk to medications.

Ideally, the workflow of PGx screening should start in the surgeon's office or preoperative evaluation clinic, prior to the scheduled surgery. This will allow adequate time for results to become available on the day of the surgery. Upon reviewing the patient's EHR on the day of surgery, the healthcare providers could be alerted to potential genetic risks to specific 


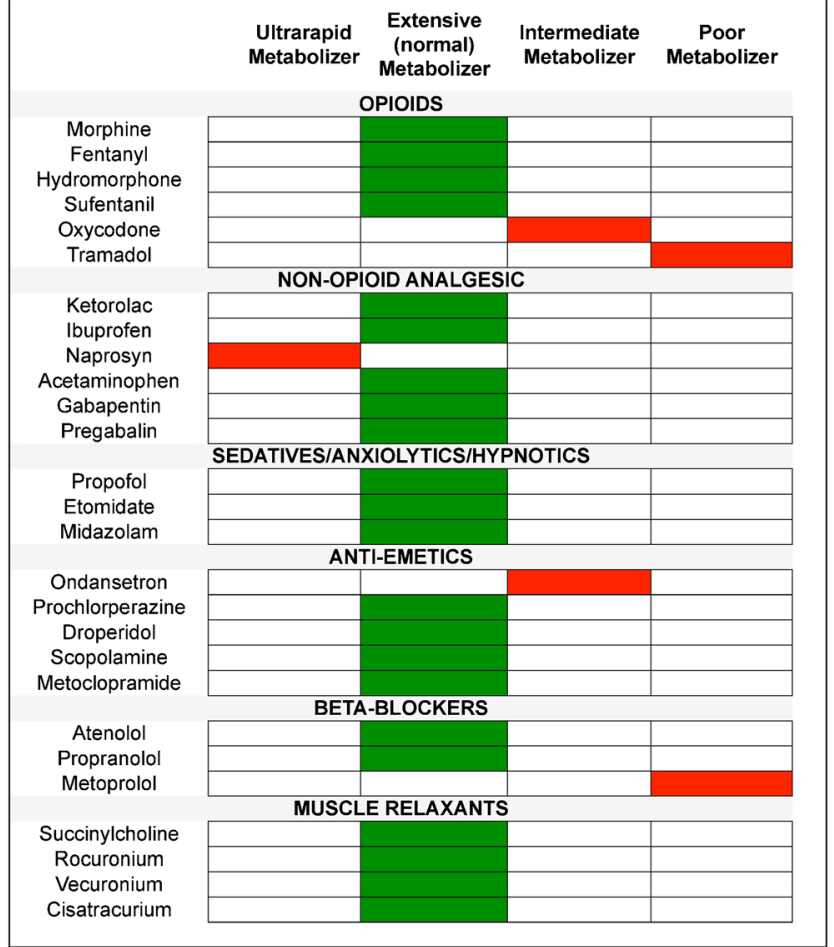

Fig. 1 Example display of the genetic risk summary to common perioperative medications for a single patient

drugs or drug classes, much like a medication or drug-to-drug interaction alerts. Furthermore, they should be able to easily view the list of genetic risks while simultaneously scanning the medical record for other clinical information. Clinical decision support tools may be integrated into the EHR as well, in which medication recommendations are created based on the patients' PGx profile. These data should be conveniently presented for perioperative providers, including anesthesiologists, nurses and surgeons in pre-, intra- and post-operative setting. Given promising data about ERAS and improved patient outcomes, integration into ERAS pathway protocols would further facilitate more widespread adoption [10, 11].

Personalized medicine holds great promise to improve perioperative outcomes. There is a plethora of possible data, including presence of many single nucleotide polymorphisms in various genes related to metabolism, that one may obtain from PGx screening of a single patient. It is, therefore, important that presentation of the results be well thought-out, strategic, useful, and well integrated into an EHR. PGx services should be adaptable and flexible when it comes to customizing these reports and should be able to work with the facility's informatics department to ease integration of PGx into regular clinical workflows. The utilization of PGx to drive perioperative systems may ultimately lead to a decrease in adverse events and hospital length of stay following major surgery.

\section{References}

1. Saba, R., Kaye, A.D., and Urman, R.D., Pharmacogenomics in anesthesia. Anesthesiol. Clin. 35(2):285, 2017.

2. Saba, R., Kaye, A.D., and Urman, R.D., Pharmacogenomics in pain management. Anesthesiol. Clin. 35(2):295, 2017.

3. Linares, O.A., Daly, D., Linares, A.D., Stefanovski, D., and Boston, R.C., Personalized oxycodone dosing: Using pharmacogenetic testing and clinical pharmacokinetics to reduce toxicity risk and increase effectiveness. Pain Med. 15(5):791, 2014.

4. Linares, O.A., Fudin, J., Schiesser, W.E., Daly Linares, A.L., and Boston, R.C., CYP2D6 phenotype-specific codeine population pharmacokinetics. J. Pain Palliat. Care Pharmacother. 29(1):4, 2015.

5. Wu, X., Yuan, L., Zuo, J., Lv, J., and Guo, T., The impact of CYP2D6 polymorphisms on the pharmacokinetics of codeine and its metabolites in Mongolian Chinese subjects. Eur. J. Clin. Pharmacol. 70(1):57, 2014.

6. Fujita, K., Ando, Y., Yamamoto, W., Miya, T., Endo, H., Sunakawa, Y., Araki, K., Kodama, K., Nagashima, F., Ichikawa, W., Narabayashi, M., Akiyama, Y., Kawara, K., Shiomi, M., Ogata, H., Iwasa, H., Okazaki, Y., Hirose, T., and Sasaki, Y., Association of UGT2B7 and ABCB1 genotypes with morphine-induced adverse drug reactions in Japanese patients with cancer. Cancer Chemother. Pharmacol. 65(2):251, 2010.

7. Takashina, Y., Naito, T., Mino, Y., Yagi, T., Ohnishi, K., and Kawakami, J., Impact of CYP3A5 and ABCB1 gene polymorphisms on fentanyl pharmacokinetics and clinical responses in cancer patients undergoing conversion to a transdermal system. Drug Metab. Pharmacokinet. 27(4):414, 2012.

8. Seripa, D., Latina, P., Fontana, A., Gravina, C., Lattanzi, M., Savino, M., Gallo, A.P., Melchionda, G., Santini, S.A., Margaglione, M., Copetti, M., di Mauro, L., Panza, F., Greco, A., and Pilotto, A., Role of CYP2D6 polymorphisms in the outcome of postoperative pain treatment. Pain Med. 16(10):2015, 2012.

9. Senagore, A.J., Champagne, B.J., Dosokey, E., Brady, J., Steele, S.R., Reynolds, H.L., Stein, S.L., and Delaney, C.P., Pharmacogenetics-guided analgesics in major abdominal surgery: Further benefits within an enhanced recovery protocol. Am. J. Surg. 213(3):467, 2017.

10. Helander, E.M., Webb, M.P., Bias, M., Whang, E.E., Kaye, A.D., and Urman, R.D., A comparison of multimodal analgesic approaches in institutional enhanced recovery after surgery protocols for colorectal surgery: Pharmacological agents. J. Laparoendosc Adv. Surg. Tech. A. 27(9):903, 2017.

11. Modesitt, S.C., Sarosiek, B.M., Trowbridge, E.R., Redick, D.L., Shah, P.M., Thiele, R.H., Tiouririne, M., and Hedrick, T.L., Enhanced recovery implementation in major gynecologic surgeries: Effect of care standardization. Obstet. Gynecol. 128(3):457, 2016. 